\title{
Experiencias y desafíos en la formación de ingenieros durante la pandemia de la covid-19
}

\section{Experiences and challenges in the training of engineers during the Covid-19 pandemic}

\author{
Díaz-Garay, Bertha Haydeé1 \\ Universidad de Lima. Lima, Perú \\ bdiaz@ulima.edu.pe \\ ORCID: https://orcid.org/0000-0002-8409-3210
}

Noriega-Araníbar, María Teresa²

Universidad de Lima. Lima, Perú

manorieg@ulima.edu.pe

ORCID: https://orcid.org/0000-0001-6824-1415

Ruiz-Ruiz, Marcos Fernando ${ }^{3}$

Universidad de Lima. Lima, Perú

mruiz@ulima.edu.pe

ORCID: https://orcid.org/0000-0001-5147-8512

Citar como: Díaz-Garay, B., Noriega-Araníbar, M. y Ruiz-Ruiz, M. (2021). Experiencias y desafíos en la formación de ingenieros durante la pandemia de la covid-19. Desde el Sur, 13(2), e0019.

\section{RESUMEN}

Dado que la educación universitaria viene cambiando a partir de la experiencia por la pandemia de la covid-19, este ensayo pretende explorar algunas experiencias y desafíos (pedagógicos, tecnológicos y administrativos) que presenta la formación de ingenieros durante la emergencia sanitaria en 2020 y 2021. A partir de una mirada sobre tres ejes fundamentales (la virtualización tecnológica del aprendizaje, la gestión de recursos y el impacto emocional del confinamiento), el ensayo discute algunas experiencias regionales y locales. Entre otros aspectos, se visibilizan realidades como la incipiente mediación tecnológica de

1 Doctora en Ciencias Contables y Empresariales por la Universidad Nacional Mayor de San Marcos. Ingeniera química, profesora e investigadora

2 Doctora en Ciencias Contables y Empresariales por la Universidad Nacional Mayor de San Marcos. Ingeniera industrial, profesora e investigadora.

3 Doctor en Ciencias de la Educación por la Pontificia Universidad Católica del Perú. Ingeniero industrial y licenciado en Educación. Profesor e investigador. 
nuestra región, la limitación de recursos para la virtualización y el acceso a la conectividad, así como los problemas generados por el confinamiento y la sobrecarga académica de los estudiantes. Si bien la discusión queda abierta, se busca proponer un espacio de reflexión docente en torno a cómo será la formación profesional de ingenieros después de la experiencia pandémica.

\section{PALABRAS CLAVE}

Enseñanza universitaria, formación en ingeniería, gestión educativa, pandemia, covid-19

\section{ABSTRACT}

Given that university education has been changing in response to the Covid-19 pandemic, this essay explores experiences and challenges (pedagogical, technological and administrative) associated with engineering education during the pandemic experience of 2020 and 2021. By focusing on three fundamental aspects -the technological virtualization of learning, resource management and the emotional impact of confinement- this essay discusses both regional and local experiences. Among other aspects, also addressed are realities such as the incipient technological mediation in our region, the limitations of resources for virtualization and access to connectivity, as well as the problems produced by confinement and the academic overload experienced by students. While the discussion remains open, we propose a space for teacher reflection on the post-pandemic nature of the professional training of engineers.

\section{KEYWORDS}

University education, engineering education, educational management, pandemic, Covid-19

\section{Introducción}

A más de un año del confinamiento obligatorio en la mayoría de países latinoamericanos, las comunidades educativas e instituciones de educación básica y superior sienten que la pandemia ha tenido un impacto agresivo en los procesos de enseñanza y de aprendizaje. Como se sabe, la Organización Mundial de la Salud (OMS) declaró el 11 de marzo de 2020 que el brote de la covid-19 se había convertido en una pandemia global. Desde entonces, la vida social cambió para toda la humanidad y se 
comenzó a vivir una de las experiencias más complicadas para el mundo. Los confinamientos forzados, las restricciones en la apertura de actividades económicas, de reunión y de movilidad, al igual que el obligatorio distanciamiento social, han afectado de forma crítica la vida cotidiana de hombres y mujeres en todo el planeta (Cucinotta y Vanelli, 2020; Ordorika, 2020).

La educación superior universitaria no ha estado exenta a esta realidad y la ha tenido que enfrentar - dependiendo del país o la regiónno siempre de igual forma ni en las mismas condiciones. Marinoni et al. (2020) exponen las características particulares a las que se han tenido que enfrentar, en este nuevo contexto y normalidad, los procesos de enseñanza y de aprendizaje en la educación superior, así como otras actividades como la investigación docente, la movilidad estudiantil y la extensión universitaria. En este escenario, la formación de ingenieros en las universidades de la región ha originado que los docentes desarrollen creativamente algunas estrategias transversales a todas las especialidades y otras tantas matizadas por la propia naturaleza de base tecnológica y científica de dicha área del conocimiento.

La pandemia de la covid-19 ha generado un punto disruptivo en la educación universitaria en general y, particularmente, en la formación de profesionales ingenieros vinculados a la ciencia y tecnología. Asimismo, el impacto no ha sido igual en países de bajos ingresos y brechas digitales, como el Perú o Argentina, donde la gestión educativa afronta sus propias contrariedades (Dekun et al., 2020; Jordan et al., 2021). En Colombia, por ejemplo, la problemática de esta «nueva educación superior» se ha reflejado en la escasez de recursos, tanto tecnológicos como de conectividad, con los estudiantes. Los docentes no necesariamente cuentan con la capacitación necesaria para afrontar esta coyuntura. No obstante la situación negativa, muchos ven cosas positivas en esta nueva educación y una oportunidad de mejora en sus instituciones (Manrique, 2020).

En Chile, por su lado, emergen con notoria visibilidad, en el nivel terciario de enseñanza, percepciones del estudiantado referidas a las brechas de competencias digitales de los docentes que afectarían la calidad de las clases online. Entre otras cosas, se advierte una desigual disponibilidad y acceso a materiales de aprendizaje e insumos computacionales, así como problemas de acceso tecnológico de Internet y de acceso económico de las familias para financiar aranceles y nuevos gastos derivados de la necesidad por contratar servicios de conectividad. Todo esto se suma a las inequidades clásicas que ya enfrentaban las universidades poco antes de la pandemia (Quintana Avello, 2020). 
Según el Banco Interamericano de Desarrollo (BID, 2020), los desafíos más destacados para la educación superior en tiempos de la covid-19 lo constituyen la inequidad en la construcción expedita de una infraestructura tecnológica, la carencia de instrumentos de evaluación o acreditación de los saberes del estudiante en un contexto de enseñanza virtual, los pocos profesores capacitados para la teleeducación, la brecha digital y el acceso limitado a las tecnologías por parte de los estudiantes. Otros desafíos que se han enfrentado según el organismo internacional son los efectos psicológicos del confinamiento (que impacta la capacidad de aprendizaje de los estudiantes), la paralización de la investigación en el contexto de la pandemia, el riesgo de la sostenibilidad financiera y el riesgo de la salud económica de las universidades.

Los países han dado diversas directivas a fin de asegurar la continuidad de la actividad académica. A modo de ejemplo, la Organización de las Naciones Unidas para la Educación, la Ciencia y la Cultura (Unesco, 2020) menciona que la Superintendencia Nacional para la Educación Universitaria del Perú (Sunedu) supervisa las clases virtuales de universidades y recomienda medidas como el desarrollo de adaptaciones no presenciales de las asignaturas de sus programas académicos, la facilitación de recursos tecnológicos, la adaptación a la forma no presencial de las evaluaciones previstas, la definición de la ruta de aprendizaje de las asignaturas - adaptándolas a mecanismos de educación no presencial- y la implementación de estrategias de acompañamiento a los estudiantes a través de docentes, tutores o guías.

Vega-González (2013) manifiesta que los profesionales de la ingeniería siempre han estado en el centro de los procesos de innovación tecnológica, económica y social. Tal vez nunca como hoy la ingeniería ha sido tan importante ante la emergencia, al responder ante la necesidad del uso y la difusión de las tecnologías de la información, de la robótica y de la biotecnología, entre muchas otras innovaciones y descubrimientos científicos que afectan todas las áreas de la vida. Los desafíos de la pandemia de la covid-19 obligaron a las carreras de ingeniería a cambiar más rápido que nunca. El principal desafío, aún por resolver, es enseñar a los futuros ingenieros a trabajar y operar hardware real no disponible en casa. Las nuevas tecnologías deben aportar a la educación en ingeniería no solo para la mejora de su calidad, sino para agilizar su aplicación en el entorno industrial. Desde este punto de vista, la pandemia constituye una oportunidad para que el mundo académico se adapte a estos cambios rápidos y versátiles (Rassudoy y Korunets, 2020).

Otro aspecto que también debe revisarse son los espacios físicos de los profesores y si estos son adecuados a los procesos de enseñanza y de 
aprendizaje. Kelly (2020) estudia el valor e impacto de dichos espacios de trabajo físico y el valor de la tecnología para los trabajadores individuales que normalmente no han trabajado desde casa y que han respondido y cambiado sus actitudes sobre dichos lugares de trabajo. Por el lado de los estudiantes, sin embargo, se observa un entusiasmo por la tecnología. Un hallazgo notable de Won et al. (2020) fue el deseo de los estudiantes de socializar y de tener interacciones mínimas en diferentes tipos de entornos virtuales, a fin de promover la sensación de unión con compañeros o profesores. La formación en clases de grupos más pequeños para facilitar tal interacción fue una estrategia exitosa. Los viajes en campos virtuales también ofrecieron una oportunidad a los profesores invitados para conectarse con la clase desde cualquier parte del mundo.

A pesar de todo, en la actualidad persiste todavía gran incertidumbre en torno al curso de la pandemia por las nuevas oleadas del virus y rebrotes. Esta situación ha provocado el cierre de centros educativos en general. Los que quedan deberán adaptarse a un modelo híbrido para continuar proporcionando educación a los estudiantes que aún se encuentran aislados (Jordan et al., 2021). Frente a este contexto, resulta pertinente seguir reflexionando en torno a las dificultades afrontadas en la educación universitaria durante este periodo -y más específicamente desde la mirada de la ingeniería, la ciencia y la tecnología-, a fin de vislumbrar los retos que se avecinan y las oportunidades que este contexto dinámico presenta para los docentes.

\section{Formando ingenieros durante la pandemia: tres pilares de reflexión}

Grandes desafíos se han generado a raíz del cambio disruptivo de la educación presencial y tradicional hacia una educación virtual que conlleva un rápido cambio del paradigma vinculado a la importancia de la relación entre el docente y el estudiante. Especialmente, a través de un acompañamiento sincrónico de todas las actividades orientadas a afianzar su desarrollo cognitivo y emocional. Los docentes han necesitado, en primer lugar, hacer un análisis reflexivo sobre su función formativa con un aula virtual en la que ahora es necesario concientizarse de que la nueva comunicación exige no solo la construcción de competencias y cimentar conocimientos, sino también de emociones, de la pasión por enseñar a través de la modulación de la voz, de la expresión del rostro y de la actitud corporal que los estudiantes logran visualizar por la pantalla.

Hardiyanta et al. (2021) concluyeron que, para lograr los objetivos de la educación profesional, se necesitan procesos de aprendizaje de calidad y recursos humanos que sean capaces de brindar buenos servicios, 
especialmente en talleres y laboratorios. En su investigación, los autores comentan que las aptitudes necesarias para sobrevivir en la era de la Revolución Industrial 4.0 incluyen competencias altamente técnicas, así como capacidades en tecnologías de la información, en creatividad e innovación, en resolución de problemas y en habilidades blandas. Estas y otras habilidades son fundamentales para desarrollar el pensamiento crítico a través de la investigación, pero siempre con el apoyo de los docentes (Cangalaya Sevillano, 2020). Sin embargo, como ya se ha mencionado, la pandemia de la covid-19 que afecta al mundo ha planteado desafíos importantes para la educación superior a nivel global. El principal desafío, que es urgente e inevitable, son las clases en la universidad impartidas en modalidad online.

En este contexto existen cinco aspectos que juegan un papel preponderante en el mencionado aprendizaje en línea. Primero, una instalación confiable de la tecnología de la información para el apoyo del acceso al aprendizaje en línea. En segundo lugar, la necesidad de que los profesores realicen variaciones e innovaciones para entregar material didáctico, de modo que atraigan a los estudiantes. En tercer lugar, se necesita un estudio y capacitación a profundidad para las conferencias prácticas, ya que los profesores pueden hacerlas recurriendo a simulaciones. En cuarto lugar, es necesario que el material se entregue sobre la base de principios ligeros, claros, concisos e interactivos. Finalmente, se advierte también un aumento en la participación de los estudiantes durante el aprendizaje en línea (Sunardiyo, 2021).

Una interrogante que se generó y despertó en su momento alarma entre los docentes de las carreras de ingeniería fue la no presencialidad en los laboratorios, tradicionalmente la estrategia de las especializaciones en las que se reconoce la necesidad de fomentar el ingenio del estudiante para proponer soluciones prácticas interactuando con fenómenos físicos, químicos, eléctricos y con procesos tecnológicos en general. El cómo superar este impasse en una educación virtual fue un tema retador que, con mayor o menor efectividad, los docentes fueron (y están) superando mientras identifican herramientas tecnológicas básicas como videos tutoriales, simuladores y animaciones, así como herramientas más avanzadas como plataformas interactivas. El uso de la realidad aumentada sigue impulsando de manera exponencial la creatividad de los profesores.

La enseñanza en ingeniería se desarrolla entonces, y dentro de este contexto, con un componente eminentemente práctico. Es importante que el proceso educativo se caracterice por ser interactivo y colaborativo, bajo la orientación del profesor, con la utilización de métodos y procedimientos que permitan al profesional en formación anticiparse e interactuar 
con la realidad y brindar soluciones en su entorno social (Capote et al., 2016). En tal sentido, si bien la formación universitaria de ingenieros en estos meses caracterizados por la emergencia sanitaria comparte aristas comunes con otras tantas especialidades, existen tres pilares que han sido críticos y determinantes en esta área del conocimiento y sobre los cuales conviene reflexionar: la virtualización y el uso de la tecnología para el aprendizaje, la gestión de recursos para el proceso formativo, y el impacto generado por el confinamiento en discentes y docentes.

\section{Virtualización pedagógica y uso de tecnologías para el aprendizaje}

Como respuesta al brote de la covid-19, se han generado oportunidades para la utilización de algunos métodos innovadores de enseñanza aprovechados en las carreras de ingeniería. Zhang Ping et al. (2020), por ejemplo, señalan que muchas clases tradicionales se han reemplazado por un proceso formativo en línea apoyado en el uso del método de aprendizaje basado en problemas (ABP). Así, los maestros guían, comprueban y gestionan el aprendizaje de sus estudiantes a través de la virtualización tecnológica y mediante diferentes tipos de problemas o preguntas de investigación. Mientras tanto, los estudiantes cimientan conocimientos y construyen competencias respondiendo y resolviendo situaciones problemáticas de forma sincrónica o asincrónica.

Para el caso de los estudiantes de ingeniería, ha sido indispensable repensar las sesiones sincrónicas para talleres, laboratorios y ejercicios de evaluación continua. Sánchez-Prieto et al. (2020) cuentan la experiencia de una asignatura para ingenieros en la que recurrieron a plataformas como el Google Meet y el Blackboard Collaborate, en reemplazo de la tradicional participación activa con sesiones en foros virtuales o videos de resolución de problemas de modelado por parte del estudiantado.

La construcción de conocimiento en esta coyuntura se ha desarrollado mucho a través de videos, lo que ha permitido que los estudiantes visualicen ensayos y experimentos comprobando empíricamente los conceptos ya recibidos. También se ha hecho uso de la conexión sincrónica con los técnicos de los laboratorios para el desarrollo de experimentos y la explicación de los instrumentos o materiales de la experiencia. La generación de datos de laboratorio de forma sincrónica, remitida a los estudiantes a fin de que realicen cálculos y análisis de resultados con el uso de softwares que permiten volver a simular las experiencias, ha permitido suplir las limitaciones de la coyuntura en este tipo de experiencias. Este ambiente virtual, que ha generado una forma diferente de relacionamiento entre docentes y estudiantes ( $y$ entre ellos mismos), permite desarrollar a la par valores como el trabajo en equipo, el respeto a los demás dentro del 
espacio virtual y la solidaridad ante la necesidad colectiva, compartiendo datos o incluso conexión a Internet de forma creativa.

Paralelamente, $y$ en referencia al proceso evaluativo, afrontar una evaluación online masiva es algo a lo que las universidades, bajo el tradicional esquema de la presencialidad, no se habían enfrentado seriamente hasta ahora desde una perspectiva institucional. El profesorado y estudiantado, por tanto, tienen que colaborar para dar una respuesta que integre decisiones metodológicas y tecnológicas, a la vez que garanticen la equidad, la seguridad jurídica y la transparencia para todos los actores, internos y externos (Fardoun et al., 2020; García-Peñalvo et al., 2020). Diseñar una evaluación online demanda ciertas consideraciones, sobre todo en el caso de estudiantes de ingeniería.

Desde luego, no se trata de replicar las tradicionales evaluaciones presenciales y simplemente colocarlas en formato virtual (García-Peñalvo et al., 2019). Sánchez-Prieto et al. (2020) prescinden del elemento sorpresa tradicional para el modelado de algoritmos durante el proceso de evaluación. Los autores comentan que una ventaja del nuevo formato es que permite entregar el enunciado a modelar en sesiones sincrónicas previas. La resolución del problema de modelado en ingeniería tiene la ventaja que resulta casi imposible que dos estudiantes propongan la misma solución empleando los mismos términos conceptuales.

La naturaleza del modelado en ingeniería ha permitido que muchas veces se pueda prescindir de un sistema de supervisión remota a través de cámaras, también llamado e-proctoring (González-González et al., 2020). La utilidad de esta herramienta de monitoreo por cámara, a diferencia de las evaluaciones en otros contextos formativos, viene dada para la calificación de conceptos teóricos. Un ejemplo de lo anteriormente indicado es el Respondus LockDown Browser, herramienta cuya pertinencia y vulnerabilidad (Araujo-Novoa, 2020) deben ser cuidadosamente analizadas para las pruebas en ciertas materias de ingeniería. El desarrollo de situaciones problemáticas que demanden el acceso simultáneo a otros recursos (web sites, calculadoras, simuladores online, archivos y formularios, etc.) dificultan su empleo. Adicionalmente, otra de las principales dificultades ya aludidas en este sentido es la heterogeneidad de acceso y de disponibilidad de accesorios de hardware por parte de algunos estudiantes de ingeniería, en la que debe primar el criterio de equidad en el proceso evaluativo.

Pese a lo anteriormente señalado, el alcance de las tecnologías que facilita los procesos de enseñanza, de aprendizaje y de evaluación no se difunde suficientemente en todos los contextos para superar la posible deserción estudiantil. Para los docentes investigadores, incluso, se omite 
la visibilidad de estrategias que garanticen su continuidad investigativa, lo que genera paralización o retraso, e incide en el avance científico-tecnológico. Por tanto, es necesario trascender del uso exclusivo de tecnologías para docencia e impulsar la difusión sobre accesibilidad, visibilidad y capacidad de la interoperabilidad de las plataformas tecnológicas que responda a requerimientos de un entorno educativo vulnerado por la covid-19 (Paredes-Chacín, 2020).

\section{La gestión de los recursos para la formación de ingenieros durante la pandemia}

Se focaliza la gestión de recursos en la necesidad tecnológica de conectividad, tanto de docentes como de estudiantes, así como en la propia gestión del docente (como recurso humano) que debe hacer uso de la tecnología digital. En ese sentido, para mantener a su población estudiantil y lograr la participación de todos los estudiantes con la mejor calidad, las universidades han tenido que aplicar y gestionar nuevas estrategias, al proporcionar equipos y dispositivos para conectividad y al tratar de dar mayor cobertura y bienestar a su comunidad. No solo se han visto obligadas a asumir la nueva modalidad de la virtualidad. También se han visto forzadas a velar por la conectividad de sus miembros (docentes y estudiantes), aunque en algunos casos no se ha podido evitar inequidad, deserción y baja matricula, entre otras dificultades.

Lasfacultades de ingeniería asumen, en estecontexto, un reto particular: deben predicar desde el ejemplo. Se deben desarrollar contenidos virtuales asincrónicos y dinámicos. Deben disminuir, de acuerdo con la naturaleza formativa, la dependencia de lo presencial, incluso de lo virtual sincrónico. Además, deben desarrollarse aplicativos (apps) y laboratorios remotos con simuladores, a los que los estudiantes accedan en cualquier momento desde sus teléfonos móviles y computadores (Giordano et al., 2020).

En contraste, los estudiantes, algunas veces de forma mayoritaria, no valoran positivamente el paso a la virtualidad, porque advierten un incremento de la carga lectiva. La formación a distancia y online ha contribuido al impacto negativo en la visión de un sector del alumnado sobre sus formadores, mientras que estos últimos demandan en los jóvenes universitarios competencias digitales básicas, especialmente en los ingenieros en formación. Los docentes, en el escenario de la formación a distancia, deben ahora mostrar conocimientos sobre el contenido de la materia y también conocimientos tecnológicos y pedagógicos digitales. Deben ser constantemente capacitados para ser competentes en su desempeño, resolver conflictos y crear espacios de comunicación con sentido crítico (Valcazar Montenegro, 2019). 
Especialmente en el caso de los docentes ingenieros que manejan simuladores o laboratorios y recursos virtuales, existe la obligación de ser capaces de innovar, reflexionar y transformar tecnológicamente sus propuestas didácticas para responder a las demandas sociales que vive el mundo en medio de una crisis sanitaria. A la vez, deben velar porque se alcancen los objetivos curriculares propuestos al inicio del curso (Tejedor, 2020). Las universidades tienen la tarea pendiente de revisar y mejorar cómo impartir sus clases online, al recurrir a sus entornos virtuales de aprendizaje para que en un futuro pospandémico accedan más personas a la educación superior.

\section{Impacto emocional del confinamiento en docentes y estudiantes de ingeniería}

La pandemia ha generado otro gran reto para la enseñanza universitaria, particularmente porque, como lo indican Herstatt y Tiwari (2020), las personas se han sentido extraídas de sus rutinas durante semanas debido al confinamiento y han tenido que reorganizar sus actividades. Los autores reflexionan en torno a que todos los agentes involucrados en diversas actividades académicas han tenido ocasión, incluso, de recapacitar sobre preguntas vinculadas a qué es lo verdaderamente importante en la vida. Este cambio de perspectiva ontológica genera también un nuevo enfoque si se quiere lograr un aprendizaje significativo con los estudiantes y abre a la exigencia de nuevas estrategias de acompañamiento de ellos.

Los ingenieros en formación y sus docentes no están ajenos a esta realidad, compartida por estudiantes de todas las especialidades en la región y alrededor del mundo. En este sentido, una investigación abordó el tema de la salud mental en jóvenes universitarios peruanos, de 19 a 25 años, y su creciente deterioro durante la pandemia de la covid-19. Los investigadores concluyeron y reflexionaron, luego del diagnóstico, sobre la existencia de un estigma en torno al pedido de ayuda, la expresión de emociones, la vulnerabilidad y el autocuidado, los cuales, desgraciadamente, no siempre previenen a los estudiantes de pedir ayuda para atender su salud mental (Apaza et al., 2021). Otra investigación, también para el caso peruano, confirmó que una mayor ansiedad por la covid-19 se relacionaba directamente con un empeoramiento de la salud mental de 356 estudiantes universitarios (Vivanco-Vidal et al., 2020).

Rodríguez (2020), por su parte, advirtió que las mujeres universitarias presentan puntuaciones más elevadas en los factores asociados al miedo y depresión que los hombres ante la enfermedad, y concluye que el peligro de contagio está muy presente y emparentado con muertes masivas de personas, dado que se ponen en movimiento mecanismos fisiológicos 
que alertan sobre posibles daños a la vida misma. Un mensaje electrónico solicitando ayuda o apoyo ante la pérdida de un progenitor - o comprender que es difícil la situación ante la ausencia de un padre porque está internado en el hospital y debe hacerse cargo de los hermanos menores - son algunas de las situaciones que se han tenido que contemplar para ser más flexibles y considerar nuevas estrategias durante el proceso de enseñanza y de aprendizaje, como en lo referente a las evaluaciones de estos estudiantes.

Otros estudios, realizados también en los últimos años, dan cuenta del estrés asociado especialmente en estudiantes de ciencias e ingeniería que incluso acarrean situaciones médicas (Flores-Aguilar et al., 2020). Suárez y Ramírez-Díaz (2020) encontraron indicios de estrés académico en dos poblaciones de estudiantes de ingeniería en Colombia y México. Si bien el estrés estaba asociado a la evaluación en una materia específica de la carrera, cada población mostraba causas con particularidades distintas. No obstante, la sobrecarga académica en estudiantes de ingeniería es otro de los factores de estrés, junto a la falta de tiempo para cumplir con las obligaciones encargadas por sus tutores (West et al., 2021).

Esta situación, al parecer, se ha incrementado para los estudiantes en el contexto de pandemia (Tejedor et al., 2020) y, consecuentemente, para los ingenieros en formación, en un entorno tecnológico y de virtualidad que les significa una sobrecarga académica no prevista. En efecto, ha surgido un síndrome experimentado al sentirse abrumado por recibir información a través de plataformas educativas, aplicaciones móviles y correos electrónicos. A los estudiantes más vulnerables que participan en programas de nivelación y de apoyo, el aislamiento social los golpea aún más fuerte (Pedró, 2020).

Sin embargo, los propios estudiantes y docentes pueden reconocer algunas ventajas que el confinamiento también ha traído en cuanto a una rutina menos agitada, pero solo en ciertos aspectos. Algunos universitarios, por ejemplo, no quieren volver a la anterior normalidad, porque ello significa un gran número de horas perdidas en trasladarse de casa a la universidad y viceversa, una gran contaminación en el ambiente, un mayor tráfico y exponerse a la delincuencia (Sanabria y Aquino, 2020; Apaza et al., 2021).

Otros tantos han visto en este contexto una valiosa oportunidad para cursar más asignaturas o culminar su tesis de investigación con mayor tranquilidad emocional. En este sentido, la educación universitaria no debe perder su perspectiva de interdependencia con otras disciplinas - como la psicológica, biológica o antropológica-, a fin de abordarla desde una dimensión holística e integral (Loreto Mola-Olate, 2020). Esta 
situación merece, no obstante, un análisis a futuro más detallado, a fin de insertar adecuadamente a la comunidad académica en lo que será el impacto emocional de la universidad post pandemia.

\section{Conclusiones}

La formación de ingenieros comparte con otras especialidades algunas problemáticas similares en cuanto a retos y desafíos durante el periodo de pandemia, aunque con matices diferentes mediados por el tema tecnológico. Principalmente se destacan tres aspectos de reflexión cuyo análisis no se agota con el presente ensayo: la virtualización tecnológica del aprendizaje, la gestión de recursos y el impacto emocional del confinamiento para estudiantes y docentes en las carreras de ingeniería.

La virtualización tecnológica del aprendizaje ha generado, desde un inicio, que el alcance de las tecnologías de la información que facilita los procesos de enseñanza, de aprendizaje y de evaluación de ingenieros no sea comprendido totalmente por los docentes, ni se difunda suficientemente su efectividad para superar la posible deserción estudiantil en las universidades. En efecto, no todas las instituciones educativas están preparadas para ofrecer una educación virtual de calidad o que, cuando menos, sea equivalente a su oferta educativa presencial.

Por otro lado, es importante tomar atención sobre el nivel de conectividad que ofrecen los sistemas de comunicación de los diversos países en Latinoamérica, donde las brechas sociales y económicas son notables. Los Estados deben trabajar por garantizar una amplia cobertura del Internet y demás recursos tecnológicos, a fin de que lleguen a todas las poblaciones para atender las necesidades de educación de los jóvenes de nuestra región.

La covid-19 ha generado un gran impacto emocional debido al confinamiento de docentes y estudiantes de ingeniería, que repercute seriamente en los procesos de enseñanza y de aprendizaje, y constituye una variable que se ha debido considerar para cumplir con los planes de estudios programados. Esta realidad ha evidenciado también el relego tecnológico de muchos docentes ingenieros, situación que genera una nueva barrera de comunicación de aquel docente brillante en las aulas, pero que tiene dificultades para una sesión virtual. Las universidades tienen que asistir a sus profesores con la seguridad necesaria a través de capacitaciones o sesiones de coaching educativo que los ayuden a disfrutar de la experiencia de una clase virtual y que confirme y desarrolle nuevas experiencias en su vocación docente y sus años de servicio.

Se comenta mucho que nuestra juventud pertenece a una generación digital y que para ellos resulta natural el acceso a la tecnología. No obstante, 
como seres eminentemente sociales, nuestros estudiantes requieren interactuar con sus coetáneos. El confinamiento, en efecto, los sigue limitando en sus relaciones amicales, de enamoramiento, de compañerismo y del disfrute propio de su círculo de amigos. Hoy se habla del espacio «rescatado» del hogar en el que pasan horas frente al ordenador u otro medio tecnológico para atender sus responsabilidades académicas. Las distracciones propias del entorno familiar o compartir la única red de comunicación generan no solo incomodidad, sino también ansiedad y desmotivación. La tarea de revertir todas estas dificultades constituye un reto y desafío de reflexión para los docentes, con una mirada prospectiva hacia los escenarios de formación universitaria de los ingenieros pospandemia.

\section{Contribución de autoría}

Bertha Haydeé Díaz-Garay, María Teresa Noriega-Araníbar y Marcos Fernando Ruiz-Ruiz contribuyeron en la concepción, elaboración y revisión final del trabajo.

\section{Fuente de financiamiento}

Autofinanciado.

\section{Potenciales conflictos de interés}

Ninguno. 


\section{REFERENCIAS BIBLIOGRÁFICAS}

Apaza, A., Cajavilca, R., y Saurino, V. (2021). ParaCaídas: Proyecto de Comunicación para el Desarrollo sobre Salud Mental con jóvenes universitarios de instituciones privadas en Lima Metropolitana. [Tesis de grado, Pontificia Universidad Católica del Perú]. http://tesis.pucp.edu.pe/repositorio/

Araujo-Novoa, D. (2020). Análisis del uso y solución de posibles vulnerabilidades de la herramienta Safe Exam Browser para la realización de exámenes en línea. [Tesis de grado, Universidad de Vigo]. https://cutt.ly/SvfLqT9

Banco Interamericano de Desarrollo, BID. (2020). La educación superior en tiempos de Covid-19. Aportes de la Segunda Reunión del Dialogo Virtual con Rectores de Universidades Lideres de América Latina. http://dx.doi. org/10.18235/0002481

Cangalaya Sevillano, L. M. (2020). Habilidades del pensamiento crítico en estudiantes universitarios a través de la investigación. Desde el Sur, 12(1), 141-153. https://revistas.cientifica.edu.pe/index.php/desdeelsur/article/ view/595

Capote, G., Rizo, N., y Bravo, G. (2016). La formación de ingenieros en la actualidad. Una explicación necesaria. Universidad y Sociedad. Revista Científica de la Universidad de Cienfuegos, 8(1), 21-28. http://scielo.sld.cu/scielo. php?script=sci_arttext\&pid=S2218-36202016000100004

Cucinotta, D. y Vanelli, M. (2020). WHO Declares COVID-19 a Pandemic. Acta Biomed, 91(1), 157-160. https://doi.org/10.23750/abm.v91i1.9397

Dekun, M. C., Sosa, A. H., Clauser, C. F., Carreras, G. G. G. y Corrado, L. J. (2020, octubre). Estudiar ingeniería en tiempo de pandemia: la percepción de los estudiantes. XXVIII Seminário de Iniciação Científica. Universidade Regional do Noroeste do Estado do Rio Grande do Sul (Unijuí).

Fardoun, H., González-González, C. S., Collazos, C. A. y Yousef, M. (2020). Estudio exploratorio en Iberoamérica sobre procesos de enseñanza aprendizaje y propuesta de evaluación en tiempos de pandemia. Education in the Knowledge Society, 21. https://revistas.usal.es/index.php/eks/article/ view/eks20202117

Flores-Aguilar, S. G., Arroyo-Flores, M., Montejo-Arroyo, D., Moreno-Marín, F., Torres-Capetillo, E. G. y Capetillo-Hernández, G. R. (2020). Bruxismo en estudiantes de la facultad de ingeniería. Revista Mexicana de Medicina Forense y Ciencias de la Salud, 4(S1), 70-72. https://www.medigraphic.com/ cgi-bin/new/resumen.cgi?IDARTICULO=95104

García-Peñalvo, F. J., Alarcón, H. y Domínguez, A. (2019). Active learning experiences in Engineering Education. International Journal of Engineering Education, 35(1B), 305-209. 
García-Peñalvo, F. J., Corell, A., Abella-García, V. y Grande, M. (2020). Online assessment in Higher Education in the time of COVID-19. Education in the Knowledge Society (EKS), 21(12), 1-26. https://doi.org/10.14201/eks.23086

Giordano, R., González, L., Larrondo, M. y Páez, A. (2020). Reflexiones de Académicos Latinoamericanos en Pandemia. GEDC-ACOFI-CONFEDI-LACCEI. LACCEI Ediciones. https://cutt.ly/ovtst3x

González-González, C. S., Infante-Moro, A. y Infante-Moro, C. (2020). Implementation of E-proctoring in online teaching: A study about motivational factors. Sustainability, 12(8). https://doi.org/10.3390/su12083488

Hardiyanta, R. A. P., Suyanto, W., Arifin, Z., Mujaki, A. y Saputro, R. D. A. (2021). Training needs analysis for management of facilities and infrastructure learning automotive engineering. Journal of Physics: Conference Series 1833(1), 012016. https://cutt.ly/FvBURnx

Herstatt, C. y Tiwari, R. (2020). Opportunities of frugality in the post-corona era. International Journal of Technology Management, 83(110), 1-25. https://www.econstor.eu/handle/10419/220088

Jordan, K., David, R., Phillips, T. y Pellini, A. (2021). Educación durante la crisis de COVID-19: Oportunidades y limitaciones del uso de Tecnología Educativa en países de bajos ingresos. Revista de Educación a Distancia (RED), 21(65). 1-15. https://doi.org/10.6018/red.453621

Kelly, J. A. (2020, junio). Work-in-Progress - The sudden requirement to work from home due to COVID-19 pandemic restrictions: Attitudes and changes in perceived value of physical and immersive workspaces. 2020 6th International Conference of the Immersive Learning Research Network (iLRN) (pp. 385-388). IEEE.

Loreto Mora-Olate, M. (2020). Educación como disciplina y como objeto de estudio: aportes para un debate. Desde el Sur, 12(1), 201-211. https:// revistas.cientifica.edu.pe/index.php/desdeelsur/article/view/626

Manrique, D. (2020). Educación de calidad en tiempos de pandemia. http://hdl.handle.net/10654/37261

Marinoni, G., Van't Land, H. y Jensen, T. (2020). The impact of COVID-19 on Higher Education around the world. IAU Global Survey Report. https://cutt. ly/AvtaaJH

Ordorika, I. (2020). Pandemia y educación superior. Revista de la Educación Superior, 49(194), 1-8. http://189.254.1.230/ojs/index.php/resu/article/ view/1120

Organización de las Naciones Unidas para la Educación, la Ciencia y la Cultura, Unesco. (2020, mayo). Acciones de las universidades ante el covid-19. https://www.iesalc.unesco.org/2020/05/05/acciones-de-las-universidades-ante-el-covid-19/ 
Paredes-Chacín, A., Inciarte-González, A. y Walles-Peñaloza, D. (2020). Educación superior e investigación en Latinoamérica. Transición al uso de tecnologías digitales por Covid-19. Revista de Ciencias Sociales, 26(3), 98-117. https://dialnet.unirioja.es/servlet/articulo?codigo $=7565470$

Pedró, F. (2020). COVID-19 y educación superior en América Latina y el Caribe: efectos, impactos y recomendaciones políticas. Análisis Carolina, 36(1), 1-15.

Quintana Avello, I. (2020). Covid-19 y cierre de universidades. ¿Preparados para una educación a distancia de calidad? Revista Internacional de Educación para la Justicia Social, 9(3). https://revistas.uam.es/riejs/article/ view/12232

Rassudov, L. y Korunets, A. (2020, octubre). COVID-19 Pandemic challenges for engineering education. Proceedings of 2020 XI International Conference on Electrical Power Drive Systems (ICEPDS), Saint-Petersburg.

Rodríguez, L., Carbajal, Y., Narváez, T. y Gutiérrez, R. (2020). Impacto emocional por COVID-19 en estudiantes universitarios. Un estudio comparativo. Educa UMCH Revista sobre Educación y Sociedad, 1(16), 5-22. https://doi. org/10.35756/educaumch.202016.153

Sanabria, L. y Aquino, A. (2020). Principales ventajas de la modalidad virtual en tiempos de pandemia. Revista Científica UNE, 4(1), 17-24. http:// ns2.une.edu.py:7005/journal/index.php/revista_une/article/view/86/45

Sánchez-Prieto, J. C., García-Holgado, A., Vázquez-Ingelmo, A. y GarcíaPeñalvo, F. J. (2020). Adaptación de la Asignatura Ingeniería de Software durante el Periodo de Confinamiento. https://repositorio.grial.eu/handle/ grial/2162

Suárez, O. J. y Ramírez-Díaz, M. H. (2020). Estrés académico en estudiantes que cursan asignaturas de Física en ingeniería: dos casos diferenciados en Colombia y México. Revista Científica, 39(3), 341-352. https://doi. org/10.14483/23448350.15989

Sunardiyo, S. (2021, marzo). Online learning in higher education during the Covid-19 pandemic: A case study in the Department of Electrical Engineering. Universitas Negeri Semarang. IOP Conference Series: Earth and Environmental Science, 9th Engineering International Conference, EIC 2020.

Tejedor, S., Cervi, L., Tusa, F. y Parola, A. (2020). Education in times of pandemic: reflections of students and teachers on virtual university education in Spain, Italy, and Ecuador. Revista Latina de Comunicación Social, (78), 1-21. https://doi.org/10.4185/RLCS-2020-1466 
Valcazar Montenegro, E. E. (2019). Las competencias del docente de posgrado Un estudio comparativo en cuatro maestrías especializadas desde la percepción de los estudiantes. Desde el Sur, 11(1), 191-206. https://revistas.cientifica.edu.pe/index.php/desdeelsur/article/view/505

Vega-González, L. (2013). La educación en ingeniería en el contexto global: propuesta para la formación de ingenieros en el primer cuarto del siglo XXI. Ingeniería Investigación y Tecnología, 14(2), 177-190.

Vivanco-Vidal, A., Salori-Aranibar, D., Caycho-Rodriguez, T., Carbajal-Leon, C., y Noé-Grijalva, M. (2020). Ansiedad por Covid 19 y salud mental en estudiantes universitarios. Revista de Investigación en Psicología, 23(2), 197215. https://doi.org/10.15381/rinvp.v23i2.19241

West, N. R. B., Wan, A., Morán, N., Polo, D. y Torres, E. (2021). Influencia de los estresores académicos en los niveles de estrés de los estudiantes de la Facultad de Ingeniería Industrial pertenecientes a la Universidad Tecnológica de Panamá. Revista Prisma Tecnológico, 12(1), 60-64. https://doi. org/10.33412/pri.v12.1.2798

Won, A. S., Bailey, J. O. y Yi, S. (2020, junio). Work-in-progress-learning about virtual worlds in virtual worlds: How remote learning in a pandemic can inform future teaching. 20206th International Conference of the Immersive Learning Research Network (iLRN) (pp. 377-380). IEEE.

Zhang Ping, Z., Fudog, L. y Zheng, S. (2020). Thinking and Practice of Online Teaching under COVID-19 Epidemic. 2020 2nd International Conference on Computer Science and Educational Informatization (CSEI). IEEE. 